\title{
Combined Epithelioid Trophoblastic Tumor and Contralateral Synchronous Adenocarcinoma of the Lungs in a 69-Year-Old Man
}

\author{
Ju Sik Yun ${ }^{1}$ Ga Eon Kim ${ }^{2}$ Kook Joo $\mathrm{Na}^{3}$ Sang Yun Song ${ }^{3}$ \\ ${ }^{1}$ Department of Thoracic and Cardiovascular Surgery, Chonnam \\ National University Hospital, Gwang-ju, Republic of Korea \\ 2 Department of Pathology, Chonnam National University Hospital, \\ Gwang-ju, Republic of Korea

\begin{abstract}
Address for correspondence Dr. Sang Yun Song, M.D., Department of Thoracic and Cardiovascular Surgery, Lung and Esophageal Cancer Clinic, Chonnam National University Hwasun Hospital, Jeollanamdo,
\end{abstract} \\ Hwasun, Ilsim-ri 160, Republic of Korea (e-mail: sysong@jnu.ac.kr).
}

${ }^{3}$ Lung and Esophageal Cancer Clinic, Chonnam National University Hwasun Hospital, Jeollanamdo, Republic of Korea

Thorac Cardiovasc Surg 2012;60(S2):e22-e24.

An epithelioid trophoblastic tumor (ETT) is a form of gestational trophoblastic neoplasms that arise from trophoblasts. The tumor generally occurs in women of reproductive age and has a characteristic growth pattern and immunohistochemical profile. We present an extremely rare case of a primary ETT occurring in the lungs with an adenocarcinoma in the contralateral lung as synchronous double primary lung cancers in a 69-year-old man who had no history of a remote malignancy. He underwent adjuvant radiotherapy to both lungs after a staged thoracoscopic wedge resection and was doing well without recurrence or metastasis up to last follow-up.

\section{Introduction}

Gestational trophoblastic tumors (GTTs) comprise a group of rare tumors derived from trophoblastic tissue. Histologically, GTTs are classified into hydatidiform moles, invasive moles, choriocarcinomas, placental site trophoblastic tumors (PSTTs), and epithelioid trophoblastic tumors (ETTs). Of these GTTs, ETTs are very rare and consist of intermediate trophoblastic cells that typically presents in women of reproductive age.

\section{Case Report}

A 69-year-old man who had a history of chronic obstructive pulmonary disease was referred to our center with a left upper lung nodule that had increased in size $(0.4$ to $0.9 \mathrm{~cm})$ during follow-up. A preoperative chest computed tomography (CT) performed 2 months later revealed a left upper lung nodule, which had increased to $1.1 \mathrm{~cm}$ in size, and a newly developed, $0.8-\mathrm{cm}$ right upper lobe nodule (-Fig. 1). A positron emission tomography showed increased uptake in the left upper lobe and no significant uptake in the right upper lobe. There was no abnormal uptake in other sites. Thus, we recommended surgery because of the possibility of malignancy in each nodule. Because of poor pulmonary function $\left(\mathrm{FEV}_{1}\right.$ [forced expiratory volume in $1 \mathrm{~s}$ ] $=1.37 \mathrm{~L}$, $47 \%$ of predicted normal), emphysematous changes in both lungs, as confirmed by CT, we performed a staged bilateral thoracoscopic wedge resection. The histopathologic examination confirmed that the left upper lung nodule was an adenocarcinoma, with invasion into the visceral pleura (-Fig. 2). After 8 days, wedge resection of right upper lung was performed for a presumed benign or metastatic tumor. Contrary to our expectations, the histopathologic and immunochemical findings were compatible with an ETT (-Figs. 2 and 3). The $\mathrm{K}_{\mathrm{i}}-67$ proliferation index was approximately 30 to $40 \%$ and negative for human placental lactogen. Careful examination for the abdomen and pelvis showed no specific abnormalities. The patient underwent adjuvant radiotherapy in each lung. During the 10 months of follow-up, the patient appears to be doing well without evidence of recurrence or metastasis. received

February 8, 2012

accepted after revision

March 21, 2012

published online

November 24, 2012 (c) 2012 Georg Thieme Verlag KG Stuttgart · New York
DOI http://dx.doi.org/ 10.1055/s-0032-1322613. ISSN 2194-7635. 


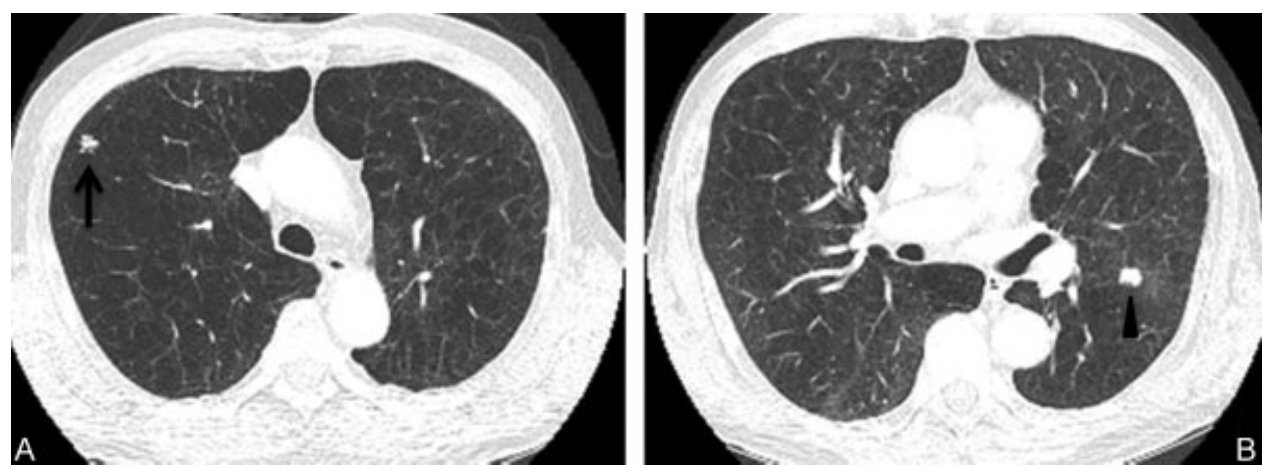

Figure 1 Preoperative chest CT scan shows separate nodules in the anterior segment of the right upper lobe (A, arrow) and apicoposterior segment of the left upper lobe ( $\mathrm{B}$, arrow head). CT, computed tomography.

\section{Discussion}

An ETT is a form of gestational trophoblastic neoplasms that arise from trophoblasts. ETTs usually occur in the uterus or endocervix, although there are some reports arising in the lungs, liver, and spine as metastatic or primary lesions, but all previously reported patients have been female. ${ }^{1}$ In 2009, a metastatic ETT in a para-aortic lymph node with a mixed germ-cell tumor of the testis was first reported in a man by Allen et al. ${ }^{2}$

The etiology of extrauterine ETTs, with the exception of metastatic lesions, is unclear. The transformation of trophoblastic cells after chemotherapy for other types of GTT has been postulated. We cannot satisfactorily explain why an ETT limited to the lungs developed in a male patient with adenocarcinoma in the contralateral lung. However, several possible theories have been suggested to explain the pathogenesis of choriocarcinoma occurring outside the gonads. ${ }^{3}$ We have reasoned that two theories may be related to our case. First, the tumor could have arisen from retained primordial germ cells that migrated abnormally during embryogenesis. Second, the tumor may have developed as a nontrophoblastic lung cancer, such as adenocarcinoma or squamous cell carcinoma and later dedifferentiated or underwent metaplasia to a trophoblast from nongonadal tissue. We also think that the possibility of a simultaneous tumor or collision tumor should be considered.
The analysis of serum hormone levels such as $\alpha$-fetoprotein and $\beta$-human chorionic gonadotropin (hCG) may be helpful in patients suspected to have ETTs. But, the definitive diagnosis requires pathologic and immunohistochemical findings. A typical histologic examination demonstrates that the tumor is composed of mononuclear intermediate trophoblastic cells. The distinctive growth pattern of ETT involves the formation of nodules, sheets, and nests with eosinophilic to clear cytoplasm. Occasionally, hemorrhage, necrosis, and lymphocytic infiltrates surrounding the tumor can occur. An immunohistochemical study is important to differentiate between trophoblastic diseases. ETTs are typically positive for cytokeratin, epithelial membrane antigen, inhibin, human placental lactogen, placental alkaline phosphatase, $\beta$-hCG, and $p 63 .{ }^{4}$ The $\mathrm{K}_{\mathrm{i}}-67$ proliferation index was 10 to $25 \%$. In our case, the specimen was histologically compatible with an ETT and nearly all of the earlier-mentioned immunohistochemical markers were positive, except for $\beta$-hCG and human placental lactogen.

Other types of GTTs, including PSTT and choriocarcinoma, must be considered in the differential diagnosis before the diagnosis of an ETT can be established. There are some differences between them in the microscopic and immunohistochemical findings. PSTTs are small, sharply circumscribed lesions, and the cells have low mitotic activity compared with ETTs. Choriocarcinomas show a dimorphic

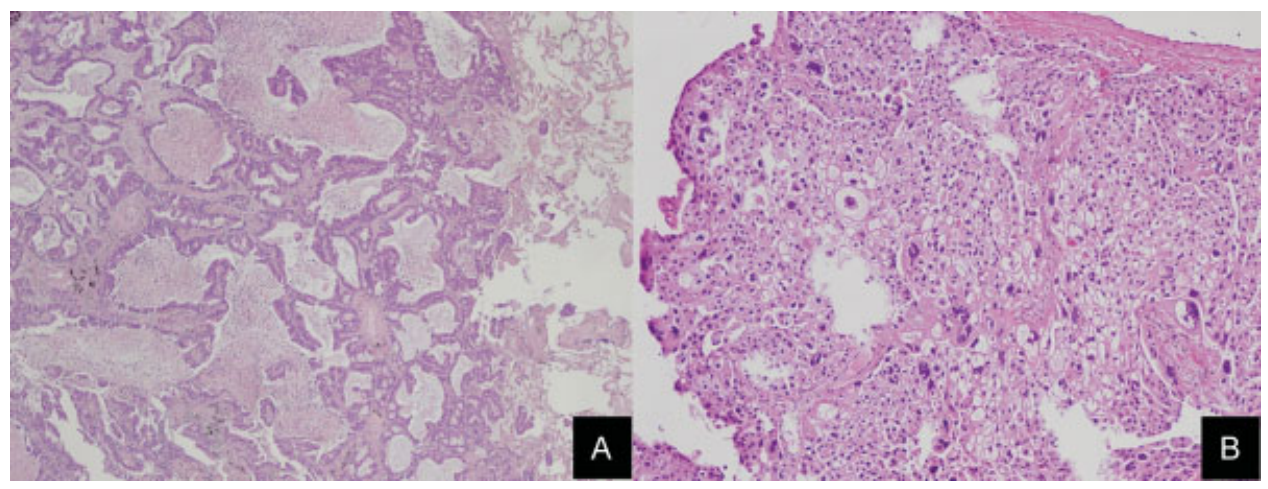

Figure 2 Pathologic findings (hematoxylin and eosin staining). (A) Left upper lobe mass. The tumor showed an acinar pattern of an adenocarcinoma, $(\times 40)$. (B) Right upper lobe mass. The tumor was composed of nested populations of predominantly mononuclear epithelioid cells, $(\times 100)$. 


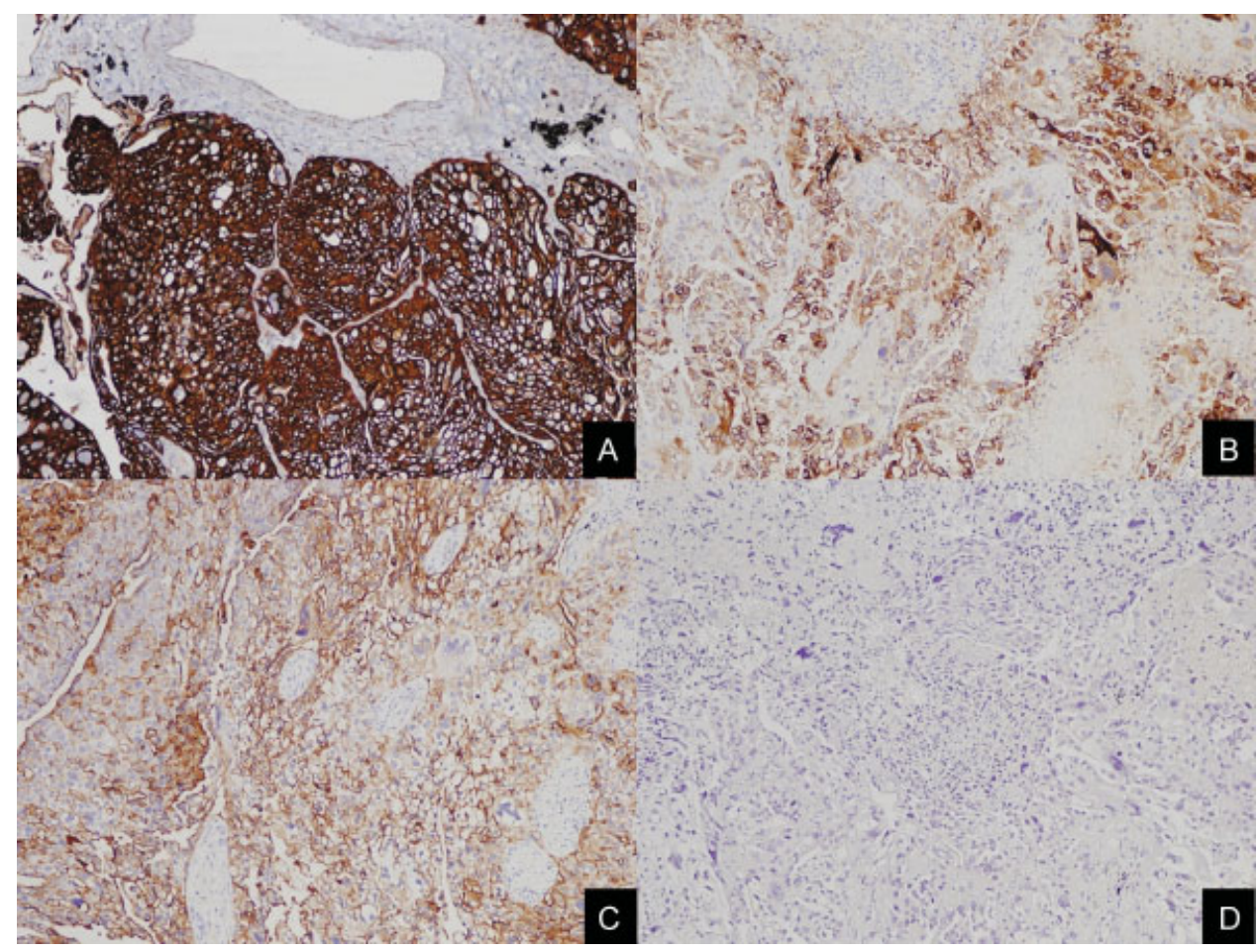

Figure 3 Immunohistochemistry of the right upper lobe mass. The tumor cells were positive for cytokeratin (A), placental alkaline phosphatase $(B)$, and CD10 (C), but negative for $\beta$-human chorionic gonadotropin (D) $(\times 100)$.

population composed of cytotrophoblastic and syncytiotrophoblastic cells growing with marked hemorrhagic necrosis. Sometimes, syncytiotrophoblastic cells can also be present in ETTs, but they are scattered and are not as prominent as in choriocarcinomas. ETTs presenting as pulmonary tumors should be distinguished from primary pulmonary carcinomas, such as squamous cell carcinomas, pleomorphic carcinomas, metastatic ETTs, and tumors of germ cell origin.

Because of the rarity of ETTs, the prognosis and clinical course are not well established. ETTs have a benign pattern of behavior, but they also have a metastatic potential. From previous reports, the metastatic and mortality rates were approximately 25 and $10 \%$, respectively. ${ }^{5}$ In most reported cases, ETTs were not responsive to chemotherapy in contrast to other types of gestational diseases. Most authors recommended surgical resection for a definitive histologic diagnosis and treatment. Thus, we reasoned that radiotherapy was a suitable adjuvant treatment option following surgical resection based on the general condition of the patient, characteristics of ETTs, and the adenocarcinoma with visceral pleural invasion on the contralateral site.

\section{Conclusion}

We have reported a patient with an unusual presentation of an ETT. This is the first case of an ETT as synchronous primary lung cancer with adenocarcinoma on the contralateral side of the lung. In addition, no remote malignancy has as yet been detected in this male patient. The diagnosis was made by pathologic and immunohistochemical studies. We believe that further clinical experience and data are needed to explain the etiology and to establish a proper treatment strategy in this situation.

\section{References}

1 Kuo KT, Chen MJ, Lin MC. Epithelioid trophoblastic tumor of the broad ligament: a case report and review of the literature. Am J Surg Pathol 2004;28(3):405-409

2 Allan RW, Algood CB, Shih IeM. Metastatic epithelioid trophoblastic tumor in a male patient with mixed germ-cell tumor of the testis. Am J Surg Pathol 2009;33(12):1902-1905

3 Kini U, Babu MK. Primary pulmonary choriocarcinoma: is it still an enigma? Indian J Chest Dis Allied Sci 2007;49:219-226

4 Shih IM, Kurman RJ. p63 expression is useful in the distinction of epithelioid trophoblastic and placental site trophoblastic tumors by profiling trophoblastic subpopulations. Am J Surg Pathol 2004;28(9):1177-1183

5 Fadare O, Parkash V, Carcangiu ML, Hui P. Epithelioid trophoblastic tumor: clinicopathological features with an emphasis on uterine cervical involvement. Mod Pathol 2006;19(1):75-82 